\title{
Method for assigning satellite lines to crystallographic sites in rare-earth crystals
}

\author{
R. L. Ahlefeldt, ${ }^{1}$ W. D. Hutchison, ${ }^{2}$ N. B. Manson, ${ }^{1}$ and M. J. Sellars ${ }^{1}$ \\ ${ }^{1}$ Centre for Quantum Computation and Communication Technology, Research School of Physics and Engineering, \\ Australian National University, Canberra, Australian Capital Territory 0200, Australia \\ ${ }^{2}$ School of Physical, Environmental and Mathematical Sciences, University of New South Wales, Canberra, \\ Australian Capital Territory 2600, Australia
}

(Received 5 July 2013; revised manuscript received 8 October 2013; published 22 November 2013)

\begin{abstract}
We describe an experimental technique for associating the satellite lines in a rare-earth optical spectrum caused by a defect with the rare-earth ions in crystal sites around that defect. This method involves measuring the hyperfine splitting caused by a magnetic dipole-dipole interaction between host ions and a magnetic defect. The method was applied to $\mathrm{Ce}^{3+}: \mathrm{EuCl}_{3} \cdot 6 \mathrm{H}_{2} \mathrm{O}$ to assign 13 of the outermost 22 satellite lines to sites. The assignments show that the optical shift of a satellite line is loosely dependent on the distance to the dopant. The interaction between host and dopant ions is purely dipole-dipole at distances greater than $7 \AA$, with an additional contribution, likely superexchange, at distances less than $7 \AA$.
\end{abstract}

DOI: 10.1103/PhysRevB.88.184424

PACS number(s): 42.50.Ex, 76.30.Kg, 61.72.S-

\section{INTRODUCTION}

The optical spectra of rare-earth ions in crystals commonly display satellite lines: multiple weak lines arrayed on either side of the optical main line and separated from it by up to terahertz. These satellite lines arise from rare-earth ions in sites neighboring point defects such as chemical impurities. Frequently, the defects causing satellite line structure in a rareearth crystal are other rare-earth impurities, and, in the case in which the optically active rare-earth ion itself is a dopant, satellite lines arise from neighboring pairs of dopant ions.

Satellite lines provide an opportunity to probe the direct neighborhood of a defect and are often used to identify, and characterize, defects in crystals. ${ }^{1-3}$ In rare-earth doped crystals, ion-pair satellite lines allow energy-transfer and resonant electronic interactions between closely separated ions to be studied. ${ }^{4}$ Because each pair site is due to ions with a fixed separation, the distance dependence of interactions can be determined from measurements on many different satellite lines. In $\operatorname{Pr}^{3+}: \mathrm{LaF}_{3}$, for example, energy transfer between $\mathrm{Pr}^{3+}$ ions separated by more than $4.2 \AA$ is predominantly due to an electric dipole-dipole interaction, ${ }^{5,6}$ but at shorter distances there is a substantial additional contribution from another mechanism, attributed to superexchange. ${ }^{5,7} \mathrm{~A}$ better understanding of these nearest-neighbor interactions is useful for practical applications, such as lasers, because even if the proportion of rare-earth ions in pair sites is small they can be the dominant source of up-conversion and fluorescence quenching processes. $^{8}$

Satellite lines have also been suggested as a way of making a frequency-addressed, ensemble-based quantum computing system that can be scaled to moderate numbers of qubits. ${ }^{9}$ This quantum computing scheme requires a crystal stoichiometric in the rare-earth ion of interest, such as $\mathrm{Eu}^{3+}$, and doped lightly with another rare earth. Satellite lines in such a material arise from $\mathrm{Eu}^{3+}$ ions surrounding dopant ions, with each satellite line due to an ensemble of $\mathrm{Eu}^{3+}$ ions in a unique position relative to the dopant ions. Using these satellite lines as qubits has the twin advantages of a high density of ions in each ensemble qubit and very strong, homogeneous interactions between qubits, which arise because the separations of ions in one qubit from their partners in another qubit are of the order of angstroms. Additionally, rare-earth ions in solids can have very long optical and nuclear coherence times, and they have long been considered a good platform for quantum computing. ${ }^{10-14}$

A common difficulty when studying interactions between rare-earth ions in satellite lines, which also hampers experimental demonstrations of quantum computing in stoichiometric materials in which these interactions are used to enact multiqubit gates, is the difficulty in determining the crystallographic site around a dopant to which a particular satellite line is due. Site assignments have only been achieved in a small number of high-symmetry materials, in which it is possible to exploit the symmetry to assign sites..$^{2,15,16}$ In this paper, we describe a method for assigning satellite lines caused by a magnetic defect to crystallographic sites that is applicable to all symmetries: satellite lines are assigned by measuring the effect of the magnetic dipole-dipole interaction between defect and rare-earth ions on the ground-state hyperfine structure of the rare-earth ion.

To demonstrate the method, site assignments were performed for satellite lines caused by $\mathrm{Ce}^{3+}$ in $\mathrm{EuCl}_{3} \cdot 6 \mathrm{H}_{2} \mathrm{O}$. Satellite lines in a rare-earth crystal such as $\mathrm{EuCl}_{3} \cdot 6 \mathrm{H}_{2} \mathrm{O}$ arise from the distortion to the crystal lattice caused by the difference in radius between the dopant and host ions. ${ }^{29}$ This means that the satellite line structure of $\mathrm{EuCl}_{3} \cdot 6 \mathrm{H}_{2} \mathrm{O}$ doped with different rare-earth ions changes only by a scaling factor dependent on the dopant radius. Therefore, satellite line assignments made for one rare-earth dopant in $\mathrm{EuCl}_{3} \cdot 6 \mathrm{H}_{2} \mathrm{O}$ can be applied to all rare-earth doped $\mathrm{EuCl}_{3} \cdot 6 \mathrm{H}_{2} \mathrm{O}$ crystals. $\mathrm{EuCl}_{3} \cdot 6 \mathrm{H}_{2} \mathrm{O}$ was chosen because it is a good candidate for the stoichiometric quantum computing scheme described above, as it can have long coherence times when fully deuterated and has the narrowest optical inhomogeneous linewidth of any stoichiometric solid, allowing a high density of ions in each prepared ensemble qubit. The site assignments presented in this paper will allow the interactions between different satellite lines to be measured and characterized and the performance of multiqubit gates enacted using these interactions to be 
estimated. These interaction measurements will be presented in a subsequent paper.

\section{THEORETICAL BACKGROUND}

$\mathrm{EuCl}_{3} \cdot 6 \mathrm{H}_{2} \mathrm{O}$ is a monoclinic crystal with $P 2 / n$ crystal symmetry and $\mathrm{C}_{2}$ symmetry at the single $\mathrm{Eu}^{3+}$ site. ${ }^{17,18}$ When $\mathrm{EuCl}_{3} \cdot 6 \mathrm{H}_{2} \mathrm{O}$ is doped with another rare earth, the dopant substitutes at the $\mathrm{Eu}^{3+}$ site. The dopant distorts the surrounding crystalline environment, shifting the optical transition frequencies of surrounding $\mathrm{Eu}^{3+}$ ions. For those $\mathrm{Eu}^{3+}$ ions close to the dopant, the perturbation is sufficiently large to shift the frequency outside the inhomogeneously broadened line, resulting in satellite lines appearing in the spectrum of the ${ }^{7} \mathrm{~F}_{0} \rightarrow{ }^{5} \mathrm{D}_{0}$ transition. The positions of the first shell of $\mathrm{Eu}^{3+}$ ions around the dopant site are shown in Fig. 1, with the sites labeled according to their distance from the dopant ion. Because the dopant site retains $\mathrm{C}_{2}$ symmetry, there are pairs of equivalent $\mathrm{Eu}^{3+}$ ions around the dopant which are crystallographically identical and contribute to the same satellite line, for instance, sites 1 and 1' in Fig. 1. The only sites for which a single ion position gives rise to a satellite line are the sites on the $\mathrm{C}_{2}$ axis, sites 2 and 3 .

The electronic interaction between host and dopant ions that leads to satellite structure is not well understood and therefore cannot be used to assign satellite lines to crystallographic sites around the dopant. Instead, in this paper we make use of the much simpler magnetic interaction between the $\mathrm{Eu}^{3+}$ nuclear spin and the large magnetic moment of a Kramers dopant.

The two $\mathrm{Eu}^{3+}$ isotopes, ${ }^{151} \mathrm{Eu}$ and ${ }^{153} \mathrm{Eu}$, have nuclear spin $I=\frac{5}{2}$, and the singlet optical ground $\left({ }^{7} \mathrm{~F}_{0}\right)$ and excited $\left({ }^{5} \mathrm{D}_{0}\right)$ states are split into three doubly degenerate hyperfine levels in

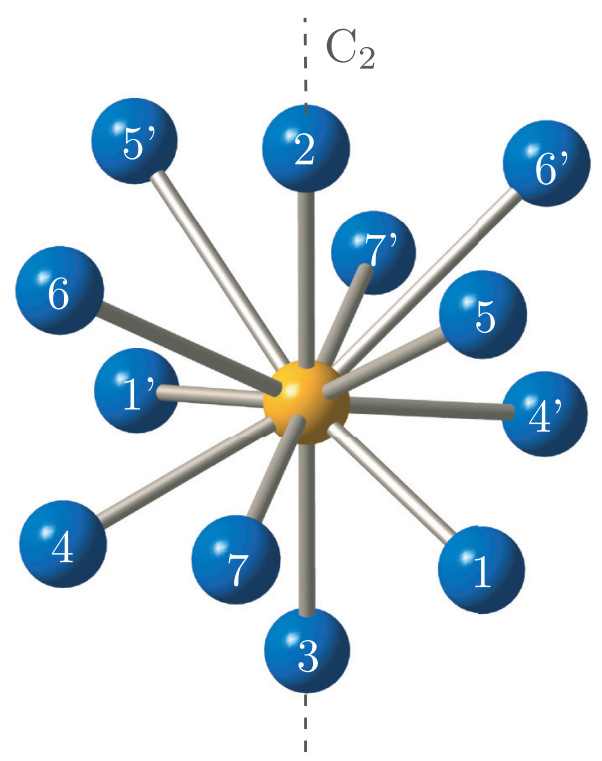

FIG. 1. (Color online) The first shell of $\mathrm{Eu}^{3+}$ ion positions around a central dopant ion in $\mathrm{EuCl}_{3} \cdot 6 \mathrm{H}_{2} \mathrm{O}$, with ion sites labeled according to their distance from the dopant. The crystal $\mathrm{C}_{2}$ axis runs through sites 2 and 3, and the dashes indicate equivalent sites related by a $180^{\circ}$ rotation about the $\mathrm{C}_{2}$. The ions are separated from the dopant by between $6.4 \AA$ (site 1) and $7.9 \AA$ (site 7). The closest ions in the next shell are $9.7 \AA$ from the dopant. zero magnetic field, with splittings of the order of $50 \mathrm{MHz} .{ }^{19}$ The hyperfine structure of a single electronic state can be described by a spin Hamiltonian of the form ${ }^{20}$

$$
H=\mathbf{B} \cdot \mathbf{M} \cdot \hat{\mathbf{I}}+\hat{\mathbf{I}} \cdot \mathbf{Q} \cdot \hat{\mathbf{I}},
$$

with $\mathbf{B}$ the magnetic field, $\hat{\mathbf{I}}$ the nuclear spin operator,

$$
M=R\left(\alpha, \beta, \gamma_{m}\right)\left[\begin{array}{lll}
g_{x} & 0 & 0 \\
0 & g_{y} & 0 \\
0 & 0 & g_{z}
\end{array}\right] R^{T}\left(\alpha, \beta, \gamma_{m}\right)
$$

the enhanced nuclear Zeeman tensor, and

$$
Q=R\left(\alpha, \beta, \gamma_{q}\right)\left[\begin{array}{lll}
-E & 0 & 0 \\
0 & E & 0 \\
0 & 0 & D
\end{array}\right] R^{T}\left(\alpha, \beta, \gamma_{q}\right)
$$

the effective quadrupole tensor. In Eqs. (2) and (3), $R(\alpha, \beta, \gamma)$ is a rotation matrix in three Euler angles, of the form given in Ref. 21. The $\mathrm{C}_{2}$ symmetry of the $\mathrm{Eu}^{3+}$ site constrains one of the three principal axes of both $\mathbf{M}$ and $\mathbf{Q}$ to lie along the crystal $\mathrm{C}_{2}$ axis. The orientation of the $\mathrm{C}_{2}$ axis relative to the $z$ axis of the coordinate system used is given by the Euler angles $\alpha$ and $\beta$. The spin Hamiltonian of the optical ground and excited states of $\mathrm{Eu}^{3+}$ in $\mathrm{EuCl}_{3} \cdot 6 \mathrm{H}_{2} \mathrm{O}$ has been characterized previously. ${ }^{19}$

To assign satellite lines to crystallographic sites in $\mathrm{EuCl}_{3} \cdot 6 \mathrm{H}_{2} \mathrm{O}$, the crystal was doped with $\mathrm{Ce}^{3+}$, a Kramers dopant. The presence of a Kramers dopant has been shown to lead to superhyperfine splitting of the ground-state hyperfine levels that differs for different satellite lines, with the size of the splitting commensurate with a magnetic dipole-dipole interaction between the electronic moment of the Kramers dopant ion and the nuclear moment of the $\mathrm{Eu}^{3+}$ ions. ${ }^{22}$ Assuming a magnetic dipole-dipole interaction, the spin Hamiltonian of the coupled $\mathrm{Ce}^{3+}-\mathrm{Eu}^{3+}$ system is the spin Hamiltonian in Eq. (1) with two additional terms, the Zeeman Hamiltonian of the dopant and the dipole-dipole interaction:

$$
H^{i}=\mathbf{B} \cdot \mathbf{M} \cdot \hat{\mathbf{I}}+\hat{\mathbf{I}} \cdot \mathbf{Q} \cdot \hat{\mathbf{I}}+\hat{\mathbf{B}} \cdot \mathbf{M}_{\mathbf{C e}} \cdot \hat{\mathbf{S}}+H_{d d}^{i} \cdot
$$

In this equation $\hat{\mathbf{S}}$ and $\mathbf{M}_{\mathbf{C e}}$ are the electron-spin operator and Zeeman tensor, respectively, of $\mathrm{Ce}^{3+}$, and $H_{d d}^{i}$ is the magnetic dipole-dipole interaction. The dipole-dipole interaction is dependent on the position of the $\mathrm{Eu}^{3+}$ ion relative to the dopant, given by the site index $i$, meaning that different satellite lines, which are due to different crystallographic sites, will have different hyperfine splittings.

The dipole-dipole interaction can be written

$$
\begin{aligned}
H_{d d}^{i}= & \frac{\mu_{0} h}{4 \pi\left|\boldsymbol{r}_{i}\right|^{3}}\left[(\mathbf{M} \cdot \hat{\mathbf{I}})\left(\mathbf{M}_{\mathrm{Ce}} \cdot \hat{\mathbf{S}}\right)\right. \\
& \left.-3\left(\hat{\boldsymbol{r}}_{\boldsymbol{i}} \cdot \mathbf{M} \cdot \hat{\mathbf{I}}\right)\left(\hat{\boldsymbol{r}}_{\boldsymbol{i}} \cdot \mathbf{M}_{\mathrm{Ce}} \cdot \hat{\mathbf{S}}\right)\right],
\end{aligned}
$$

where $\boldsymbol{r}_{\boldsymbol{i}}$ is the position vector joining the $\mathrm{Eu}^{3+}$ and $\mathrm{Ce}^{3+}$ sites, which can be determined for each site from the crystal structure. ${ }^{18}$ The Zeeman tensor $\mathbf{M}_{\mathbf{C e}}$ of $\mathrm{Ce}^{3+}$, which has the same form as Eq. (2), has been measured in $\mathrm{YCl}_{3} \cdot 6 \mathrm{H}_{2} \mathrm{O} \cdot{ }^{23}$ The Zeeman tensor components were measured to be $g_{x}=$ $37.4 \mathrm{GHz} / \mathrm{T}, g_{y}=10.9 \mathrm{GHz} / \mathrm{T}$, and $g_{z}=32.2 \mathrm{GHz} / \mathrm{T}$, with one principal axis $\left(g_{z}\right)$ lying along the crystal $\mathrm{C}_{2}$ axis, while the others lie in the (010) plane with the axis corresponding to $g_{y} 21^{\circ}$ clockwise from [100]. As $\mathrm{YCl}_{3} \cdot 6 \mathrm{H}_{2} \mathrm{O}$ is isostructural 
with $\mathrm{EuCl}_{3} \cdot 6 \mathrm{H}_{2} \mathrm{O}$ and the two rare-earth ions are fairly similar in radius, the electronic magnetic moment of $\mathrm{Ce}^{3+}$ in $\mathrm{EuCl}_{3} \cdot 6 \mathrm{H}_{2} \mathrm{O}$ will be very similar to that of $\mathrm{Ce}^{3+}$ in $\mathrm{YCl}_{3} \cdot 6 \mathrm{H}_{2} \mathrm{O}$.

\section{EXPERIMENTAL METHOD}

Satellite lines were assigned to crystallographic sites in $0.1 \% \mathrm{Ce}^{3+}: \mathrm{EuCl}_{3} \cdot 6 \mathrm{H}_{2} \mathrm{O}$ by recording the hyperfine splitting of each satellite line as an external magnetic field was rotated about the sample and fitting this rotation pattern to the spin Hamiltonian of Eq. (4) to determine the site index $i$.

The $0.1 \% \mathrm{Ce}^{3+}: \mathrm{EuCl}_{3} \cdot 6 \mathrm{H}_{2} \mathrm{O}$ crystal used was grown from a water solution using $99.999 \% \mathrm{CeCl}_{3} \cdot 7 \mathrm{H}_{2} \mathrm{O}$ and $\mathrm{EuCl}_{3} \cdot 6 \mathrm{H}_{2} \mathrm{O}$ starting materials. The 2 -mm-thick crystal was mounted in a set of three-axis superconducting coils, with the [100] axis approximately parallel to the $x$ axis of the superconducting coils and the laser direction, and the [010] direction approximately aligned with the $-z$ axis of the coils. The laser was polarized along the crystal $\mathrm{C}_{2}$ axis, [010]. The sample was cooled to approximately $2 \mathrm{~K}$ in a helium bath cryostat.

To initially locate the satellite lines, a two-dimensional Raman heterodyne ${ }^{24}$ double-resonance spectrum of $0.1 \%$ $\mathrm{Ce}^{3+}: \mathrm{EuCl}_{3} \cdot 6 \mathrm{H}_{2} \mathrm{O}$ was recorded about the $29-\mathrm{MHz}$ groundstate transition of ${ }^{151} \mathrm{Eu}^{3+}$ in zero applied field. This and subsequent rotation spectra were recorded using the optical field supplied by a Coherent 699-29 dye laser tuned to the ${ }^{7} \mathrm{~F}_{0} \rightarrow{ }^{5} \mathrm{D}_{0}$ transition at $517148.5 \mathrm{GHz}$ and the rf field supplied by a small eight-turn coil mounted around the crystal inside and coaxial with the $x$ axis of the superconducting coils. An rf signal of approximately $0.1 \mathrm{mT}$ was generated with an HP spectrum analyzer connected to a 40-W rf amplifier. The combination of optical and rf fields results in coherent emission at the sum and difference frequencies of the two fields, which was detected as a beat on the transmitted laser light by the same spectrum analyzer that was used to generate the rf field.

To build up a double-resonance spectrum, the laser was stepped through the optical line in 200 steps and individual Raman heterodyne spectra of the hyperfine lines were recorded. The resulting spectrum is shown in Fig. 2. Twodimensional spectra like this are useful because they separate satellite lines that are overlapped in optical frequency but have different hyperfine frequencies, allowing more satellite lines to be distinguished: in Fig. 2, more than 30 satellite lines can be seen. Rotation patterns were recorded for the outermost 22 lines, which are labeled in the figure.

The experimental method used for rotation patterns was similar to that reported previously. ${ }^{19}$ The hyperfine structure was recorded with Raman heterodyne spectroscopy while an external magnetic field was rotated in a spiral in 200 steps about the sample given by

$$
\mathbf{B}=\left[\begin{array}{c}
-B_{0} \sqrt{1-t^{2}} \sin 6 \pi t \\
B_{0} t \\
-B_{0} \sqrt{1-t^{2}} \cos 6 \pi t
\end{array}\right]
$$

for $t$ ranging from -1 to +1 .

Initially, rotation patterns for the 27- and 29-MHz hyperfine ground-state transitions of ${ }^{151} \mathrm{Eu}^{3+}$ were recorded for unperturbed $\mathrm{Eu}^{3+}$ ions in the center of the optical line at $517148.5 \mathrm{GHz}$, using $B_{0}=21 \mathrm{mT}$. This allowed the

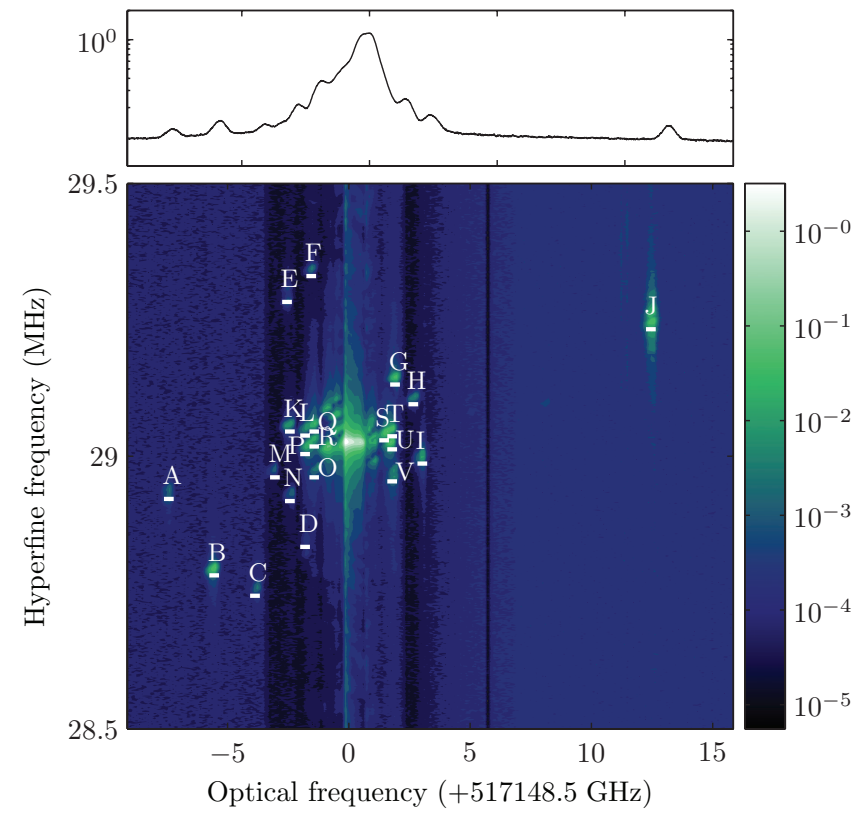

FIG. 2. (Color online) Excitation spectrum of $0.1 \% \mathrm{Ce}^{3+}$ : $\mathrm{EuCl}_{3} \cdot 6 \mathrm{H}_{2} \mathrm{O}$ with a logarithmic vertical axis (top) and doubleresonance spectrum of the 29-MHz ground-state transition of ${ }^{151} \mathrm{Eu}^{3+}$ in $0.1 \% \mathrm{Ce}^{3+}: \mathrm{EuCl}_{3} \cdot 6 \mathrm{H}_{2} \mathrm{O}$ in zero magnetic field (bottom). Both the vertical axis of the excitation spectrum and the color axis of the double-resonance spectrum give intensity in arbitrary units. The labeled satellite lines are those rotation patterns were recorded for. The color axis in this, and all subsequent double-resonance spectra, is logarithmic.

orientation of the magnet coordinate frame relative to that of the previous spin-Hamiltonian characterization ${ }^{19}$ to be determined. Following this, rotation patterns were recorded for only the 29-MHz hyperfine ground-state transition for the 22 labeled satellite lines in Fig. 2 with $B_{0}=10.5$ mT. A smaller applied magnetic field was used for the satellite lines because, at the higher field value, the magnetic dipole-dipole interaction is only a small perturbation on the strong magnetic Zeeman interaction, making it difficult to resolve.

\section{RESULTS}

Raman heterodyne-detected rotation patterns of the unperturbed main line of the ${ }^{7} \mathrm{~F}_{0} \rightarrow{ }^{5} \mathrm{D}_{0}$ transition about the 27 and $29-\mathrm{MHz}$ hyperfine ground-state transitions of ${ }^{151} \mathrm{Eu}^{3+}$ are shown in Fig. 3. The rotation patterns in Fig. 3 are described by Eq. (1), where the spin-Hamiltonian parameters are given in Ref. 19. The red lines in this figure are a fit to the data using this spin Hamiltonian. The parameters in the fit are three Euler angles $\alpha_{B}, \beta_{B}$, and $\gamma_{B}$ that describe a rotation matrix $R\left(\alpha_{B}, \beta_{B}, \gamma_{B}\right)$ that rotates the magnetic field $\mathbf{B}$ into a coordinate frame fixed with respect to the crystal axes: $([100],[100] \times[010],[0 \overline{1} 0])$. The fit shown in Fig. 3 gave $\alpha_{B}=5.00^{\circ}, \beta_{B}=7.79^{\circ}$, and $\gamma_{B}=-6.51^{\circ}$, which is reasonable given that aligning the crystal with the magnetic field coils by eye is only accurate to $\pm 10^{\circ}$. While this fit gives the alignment of the magnetic field relative to the crystal axes, there is still an uncertainty of $\delta= \pm 10^{\circ}$ in the orientation of the $\mathbf{M}$ and $\mathbf{Q}$ tensors in the (010) plane. This angle cannot 

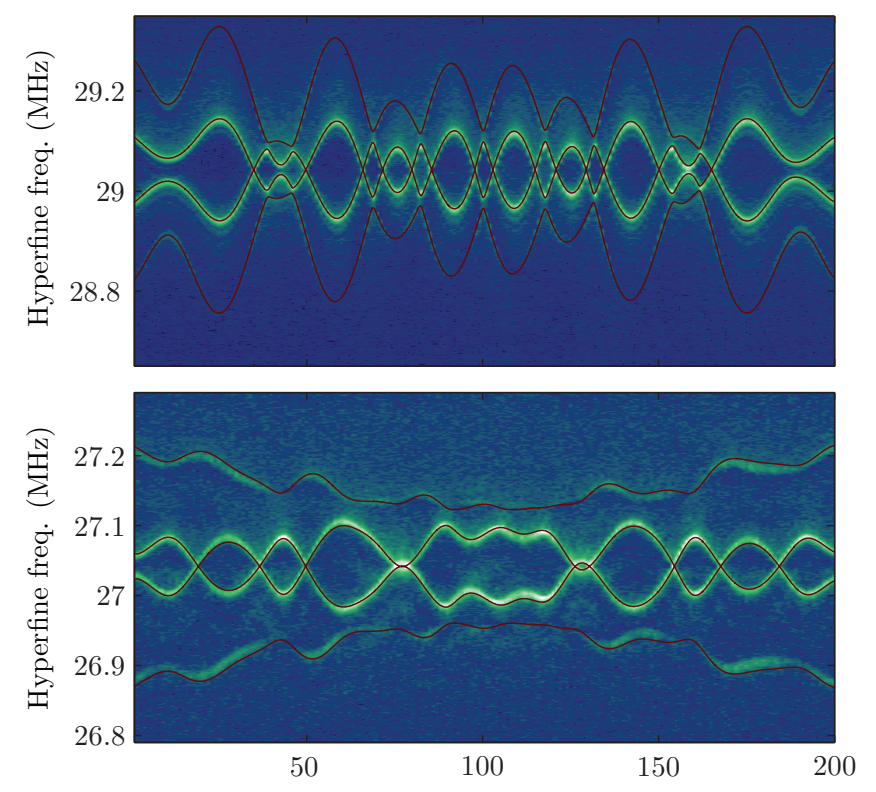

Magnetic field spiral step number

FIG. 3. (Color online) Experimental rotation pattern for the main line of $0.1 \% \mathrm{Ce}^{3+}: \mathrm{EuCl}_{3} \cdot 6 \mathrm{H}_{2} \mathrm{O}$ about the ground-state transition of ${ }^{151} \mathrm{Eu}^{3+}$. The red lines are a fit to the spectrum.

be determined from rotation patterns of the main line as it does not affect the spin Hamiltonian in Eq. (1), but it does affect rotation patterns of the satellite lines. The theoretical fits shown in the remainder of this paper use $\delta=-8^{\circ}$, as this gives the best fit to most satellite lines.

Rotation patterns were recorded for each satellite line about the 29-MHz ground-state transition of ${ }^{151} \mathrm{Eu}^{3+}$. Three example satellite line rotation patterns are shown in Figs. 4 and 5. The experimental patterns differ substantially from that of the main line, Fig. 3, with the four lines seen in that pattern split into either 8 or 16 lines in the satellite patterns by the magnetic dipole-dipole interaction with the $\mathrm{Ce}^{3+}$ dopant. For those satellite lines in which only one ion position contributes to the line (sites 2 and 3, lines $\mathrm{A}$ and $\mathrm{C}$ ), eight lines are observed in the spectrum, due to the two possible orientations of the $\mathrm{Ce}^{3+}$ pseudo-spin- $\frac{1}{2}$ magnetic moment. For most of the lines, two ion positions related by a $\mathrm{C}_{2}$ rotation about the dopant

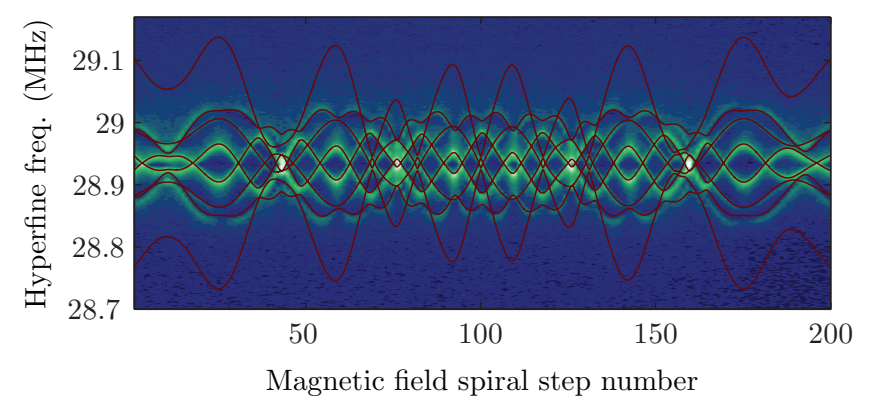

FIG. 4. (Color online) Rotation pattern of $0.1 \%$ $\mathrm{Ce}^{3+}: \mathrm{EuCl}_{3} \cdot 6 \mathrm{H}_{2} \mathrm{O}$ at $517140.95 \mathrm{THz}$ (line A). Red lines show the best-fit theoretical pattern: site 2 or 3, the two sites on either side of the dopant along the $\mathrm{C}_{2}$ axis. While these sites are crystallographically distinct, they have the same rotation patterns.

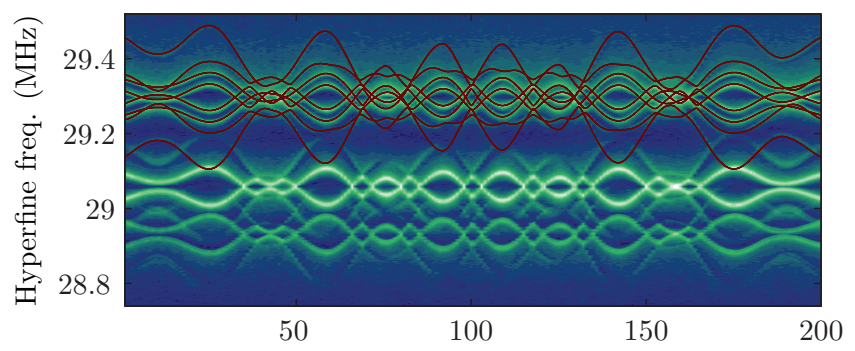

Magnetic field spiral step number

FIG. 5. (Color online) Rotation pattern of $0.1 \% \mathrm{Ce}^{3+}$ : $\mathrm{EuCl}_{3} \cdot 6 \mathrm{H}_{2} \mathrm{O}$ at $517145.79 \mathrm{THz}$ (lines $\mathrm{E}, \mathrm{K}$, and $\mathrm{N}$ ). Line $\mathrm{E}$ is assigned to site 7 .

ion site contribute to one satellite line. For these satellite lines, 16 lines can be observed in the rotation pattern because the two ion positions have different magnetic interactions with the dopant. Often, however, the splitting between the two ion positions contributing to these satellite lines is too small to be resolved in the rotation pattern, and eight lines are seen.

Also shown in Figs. 4 and 5 are the theoretical patterns for the crystallographic sites that give the best match to each experimental rotation pattern. The only differences between the main line and satellite line patterns are a small difference in zero-field hyperfine frequency, which can be seen in the double-resonance spectrum in Fig. 3, and the effect of the magnetic dipole-dipole interaction, which has one unknown parameter, the site index $i$. To fit a theoretical spectrum, it was first offset to the same rf frequency as the experimental spectrum, and then the spectra for different $i$ were compared by eye to find the best-fit site.

Satellite line $\mathrm{A}$ is due to one of the two sites on the $\mathrm{C}_{2}$ axis, sites 2 and 3 . Although these two sites are crystallographically inequivalent, they have the same rotation pattern because the two sites are the same distance from the dopant along the same axis. Satellite line E is due to site 7 , while line $\mathrm{J}$ is due to site 1 . Two other satellite lines, lines $\mathrm{N}$ and $\mathrm{K}$, are visible in Fig. 5, but these have patterns very similar to the main line and cannot be assigned to a crystallographic site. This does not necessarily imply that these lines are due to sites a long way away from the dopant: the dipole-dipole interaction is strongly dependent on the orientation of the site, and there are a number of sites at distances of less than $15 \AA$ that show almost no theoretical dipole-dipole splitting in the rotation pattern. Even some satellite lines that do show a dipole-dipole splitting can be difficult to assign to a site, as many of the outer sites (separated by more that $10 \AA$ ) have very similar patterns to each other. The site assignments that were made are summarized in Table I.

\section{DISCUSSION}

In the model used to fit the hyperfine structure of satellite lines, the magnetic interaction between host and dopant ions was assumed to be dipole-dipole. For the outer satellite lines (sites 7 and above), such as line E (Fig. 5), the dipoledipole interaction fit the data extremely well, justifying this assumption. However, for lines J (Fig. 6) and D, the fit to the data is not as good as for the outer lines, although it is sufficient 
TABLE I. Association between ion sites and satellite lines determined from experimental rotation patterns. Only those sites that could be assigned are listed. The sites are labeled by their distance from the dopant. The actual site positions can be generated from the crystal structure, ${ }^{18}$ and the positions of the nearest seven sites to the dopant ions are shown in Fig. 1.

\begin{tabular}{lll}
\hline \hline Line & Site & Distance from dopant $(\AA)$ \\
\hline A & 2 or 3 & 6.52 \\
B & 9 or 10 & 10.27 \\
C & 2 or 3 & 6.52 \\
D & 4 & 6.74 \\
E & 7 & 7.94 \\
F & 9 or 10 & 10.27 \\
G & 15 & 12.10 \\
H & 8 & 9.66 \\
I & 11 & 10.43 \\
J & 1 & 6.36 \\
L & 19 or 22 & $12.89(19), 13.27(22)$ \\
M & 16,20, or 21 & $12.63(16), 13.06(20,21)$ \\
V & 5 & 7.57 \\
\hline \hline
\end{tabular}

to definitively assign these lines to sites 1 and 4, respectively. Lines A (Fig. 4) and C, which were assigned to the two sites 2 and 3 on the dopant's $C_{2}$ axis, also do not fit as well as most other lines. The four anomalous satellite lines A, C, D, and $\mathrm{J}$ arise from the four innermost sites, with separations to the dopant of between 6.36 and $6.74 \AA$. The next closest sites (which give rise to lines $\mathrm{V}$ and $\mathrm{E}$ ) are $\approx 7.8 \AA$ away from the dopant and show no anomalous behavior. This suggests that a short-range interaction is contributing to the nearest sites. There are two possible sources of this interaction: crystal strain and exchange with the dopant. These are described below.

The strain caused by the $\mathrm{Ce}^{3+}$ dopant can affect the rotation pattern in two different ways. First, it could modify the quadrupole and Zeeman tensors of nearby $\mathrm{Eu}^{3+}$ ions. The quadrupole tensor is certainly modified to some extent, because the satellite lines have different zero-field hyperfine frequencies to the main line, but the difference is fairly small, $<0.5 \%$. Any substantial modification of the Zeeman tensor can be ruled out by looking at the rotation pattern of $\operatorname{Pr}^{3+}: \mathrm{EuCl}_{3} \cdot 6 \mathrm{H}_{2} \mathrm{O} . \mathrm{Pr}^{3+}$ has a similar radius to $\mathrm{Ce}^{3+}$ but no

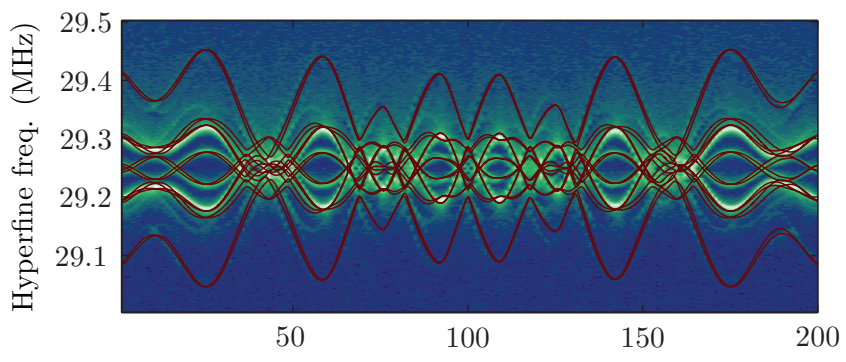

Magnetic field spiral step number

FIG. 6. (Color online) Rotation pattern of $0.1 \%$ $\mathrm{Ce}^{3+}: \mathrm{EuCl}_{3} \cdot 6 \mathrm{H}_{2} \mathrm{O}$ at $51760.67 \mathrm{THz}$ (line J). This is the outermost satellite line and corresponds to the closest ion site, site 1 . The reasons for the poor fit to this satellite line are discussed in the text. electronic magnetic moment in the ground state because the orbital angular momentum is quenched, so if the dopant was modifying the quadrupole and Zeeman tensors it would be expected that the rotation patterns on the innermost ion sites in $\mathrm{Pr}^{3+}: \mathrm{EuCl}_{3} \cdot 6 \mathrm{H}_{2} \mathrm{O}$ would be different from the main line pattern. We have measured rotation patterns for lines $\mathrm{A}$ and $\mathrm{J}$ in $\operatorname{Pr}^{3+}: \mathrm{EuCl}_{3} \cdot 6 \mathrm{H}_{2} \mathrm{O}$; both these lines have patterns identical to the main line.

The second way the dopant-induced strain can affect the rotation pattern is by shifting the positions of the surrounding $\mathrm{Eu}^{3+}$ ions from their unperturbed positions, thus altering the magnetic dipole-dipole interaction between the dopant and the $\mathrm{Eu}^{3+}$ ion. The shift of nearest-neighbor ions can be estimated by looking at the rare-earth separations in the isomorphic $\mathrm{RECl}_{3} \cdot 6 \mathrm{H}_{2} \mathrm{O}(\mathrm{RE}=\mathrm{Nd}, \mathrm{Gd}, \mathrm{Lu}){ }^{25-27}$ The rare-earth separations in these materials differ by less than $1 \%$ from that in $\mathrm{EuCl}_{3} \cdot 6 \mathrm{H}_{2} \mathrm{O}$, suggesting that a $\mathrm{Ce}^{3+}$ dopant is unlikely to shift the position of neighboring $\mathrm{Eu}^{3+}$ ions by more than $1 \%$. This is much smaller than the $10 \%$ shifts required to explain the discrepancy between experimental and theoretical rotation patterns.

The final option is an exchange interaction between the $\mathrm{Ce}^{3+}$ ion and the nearby $\mathrm{Eu}^{3+}$ ions. As the distances between the rare-earth ions are large, direct exchange is not possible, leaving superexchange via the $\mathrm{O}$ and $\mathrm{Cl}$ ligands as the only possible exchange mechanism. Superexchange has been seen between rare-earth ions in crystals at separations as large as $10 \AA .{ }^{28}$ The sharp cutoff in the interaction at $\approx 7 \AA$ does provide some evidence that the interaction is superexchange, as this is a feature of this interaction.

The site assignments presented here for $\mathrm{EuCl}_{3} \cdot 6 \mathrm{H}_{2} \mathrm{O}$ show how the optical and hyperfine frequencies of a satellite line are related to the spatial position of the ions in that line. In general, the satellite lines in $\mathrm{EuCl}_{3} \cdot 6 \mathrm{H}_{2} \mathrm{O}$ that are shifted the most in optical or hyperfine frequency are the closest sites to the dopant, but some satellite lines break this trend: line $\mathrm{B}$ has the third largest optical shift but is due to a site more than $10 \AA$ away from the dopant, while the fact that the line corresponding to site 6 (one of the first shell sites) is missing suggests that its optical shift is very small. This demonstrates that, while the interaction between dopant and host ions that results in optical and hyperfine shifts is distance dependent, the distance dependence is not strong. More concrete conclusions about the interaction and the distortion field of the dopant could be made by modeling the optical and hyperfine satellite structure using the site assignments.

While the site assignment method has been demonstrated for a stoichiometric $\mathrm{Eu}^{3+}$ crystal doped with another rare earth, it is more generally applicable. It can be used to assign satellite lines caused by any defect with a large magnetic moment. The method is useful for both low- and high-symmetry materials and can assign most of the satellite lines in any material to a single crystallographic site. In all materials with noncentrosymmetric rare-earth sites, there will be a small number of lines that can only be assigned to one of two sites related by inversion symmetry, as the spin Hamiltonian itself has inversion symmetry. This is shown here for $\mathrm{EuCl}_{3} \cdot 6 \mathrm{H}_{2} \mathrm{O}$ : lines $\mathrm{A}$ and $\mathrm{C}$ are due to sites 2 and 3, but because the two sites are related by inversion symmetry and so have identical rotation patterns it cannot be determined which line 
corresponds to which site. In centrosymmetric crystals, sites related by inversion no longer have distinct optical transition frequencies, and all sites can be assigned.

In the rare-earth doped crystals more commonly studied in rare-earth spectroscopy, site assignments could be achieved by codoping the crystal with a Kramers dopant with a similar radius. For instance, a $\operatorname{Pr}^{3+}$ doped crystal could be codoped with $\mathrm{Ce}^{3+}$. This would lead to an additional set of satellite lines due to $\mathrm{Ce}^{3+}-\mathrm{Pr}^{3+}$ pairs, which can be correlated with the $\operatorname{Pr}^{3+}-\operatorname{Pr}^{3+}$ lines because the optical shift of a satellite line is dependent on ion radius. ${ }^{29}$ The rotation pattern technique could be performed on the additional satellite lines to assign them to sites.

The measurements presented in this paper are an initial step toward using the optically resolved satellite lines as frequency addressed qubits. The criterion that allows a satellite line to be used as a qubit is that it is optically well resolved from other qubit satellite lines. Where two lines occur at the same optical frequency, one can be used. Of the 13 satellite lines whose spatial positions were determined in this paper, at least 11 could readily be used as qubits. As these satellite lines are due to the inner shells of ions around the $\mathrm{Ce}^{3+}$ dopant, the distances between the 11 qubit lines are small, ranging from 6.4 to $26.3 \AA$, and electronic interactions between the qubits, which are necessary for two-qubit gates, can be expected to be strong. The next step in this work is to measure these interaction strengths between different satellite lines.

\section{CONCLUSION}

Assigning satellite lines caused by a magnetic defect in a rare-earth crystal to the specific rare-earth ion positions around that defect is possible by utilizing the magnetic dipole-dipole interaction between the rare-earth nuclear spin and the defect electronic spin. In $\mathrm{EuCl}_{3} \cdot 6 \mathrm{H}_{2} \mathrm{O}$, this method was used to assign 13 of the 22 outermost satellite lines to crystallographic sites. These assignments showed that most of the outer satellite lines are due to the innermost site positions around the dopant. While the interaction between dopant and host ions separated by more than $7 \AA$ is wholly magnetic dipole-dipole, at distances of less than $7 \AA$, there is a small contribution to the interaction from another mechanism, likely superexchange.

\section{ACKNOWLEDGMENTS}

This research was conducted by the Australian Research Council Centre of Excellence for Quantum Computation and Communication Technology (Project No. CE110001027).
${ }^{1}$ R. L. Cone, P. C. Hansen, M. J. M. Leask, and B. Wanklyn, J. Phys.: Condens. Matter 5, 573 (1993).

${ }^{2}$ R. Jaaniso, H. Hagemann, and H. Bill, J. Chem. Phys. 101, 10323 (1994).

${ }^{3}$ M. Yamaguchi, K. Koyama, T. Suemoto, and M. Mitsunaga, Phys. Rev. B 59, 9126 (1999).

${ }^{4}$ J. Vial, R. Buisson, F. Madeore, and M. Poirier, J. Phys. (Paris) 40, 913 (1979).

${ }^{5}$ J. Vial and R. Buisson, J. Phys. Lett. (Paris) 43, 745 (1982).

${ }^{6}$ J. Hegarty, D. L. Huber, and W. M. Yen, Phys. Rev. B 25, 5638 (1982).

${ }^{7}$ G. P. Morgan, D. L. Huber, and W. M. Yen, J. Phys. Colloq. (Paris) 46, 25 (1985).

${ }^{8}$ R. J. Birgeneau, M. T. Hutchings, J. M. Baker, and J. D. Riley, J. Appl. Phys. 40, 1070 (1969).

${ }^{9}$ M. J. Sellars, E. Fraval, and J. J. Longdell, J. Lumin. 107, 150 (2004).

${ }^{10}$ G. J. Pryde, M. J. Sellars, and N. B. Manson, Phys. Rev. Lett. 84, 1152 (2000).

${ }^{11}$ M. D. Lukin and P. R. Hemmer, Phys. Rev. Lett. 84, 2818 (2000).

${ }^{12}$ K. Ichimura, Opt. Commun. 196, 119 (2001).

${ }^{13}$ M. S. Shahriar, P. R. Hemmer, S. Lloyd, P. S. Bhatia, and A. E. Craig, Phys. Rev. A 66, 032301 (2002)

${ }^{14}$ N. Ohlsson, R. Krishna Mohan, and S. Kröll, Opt. Commun. 201, 71 (2002).
${ }^{15}$ J. R. Wietfeldt and J. C. Wright, J. Chem. Phys. 83, 4210 (1985).

${ }^{16}$ J. P. Jouart, C. Bissieux, G. Mary, and M. Egee, J. Phys. C: Solid State 18, 1539 (1985).

${ }^{17}$ N. K. Bel'skii and Y. T. Struchkov, Sov. Phys. Crystallogr. 10, 15 (1965).

${ }^{18}$ D. Kepert, J. Patrick, and A. White, Aust. J. Chem. 36, 477 (1983).

${ }^{19}$ R. Ahlefeldt, M. Zhong, J. Bartholomew, and M. Sellars, J. Lumin. 143, 193 (2013).

${ }^{20}$ R. M. Macfarlane and R. M. Shelby, in Spectroscopy of Solids Containing Rare Earth Ions, edited by A. A. Kaplyanskii and R. M. Macfarlane (North-Holland, Amsterdam, 1987).

${ }^{21}$ J. J. Longdell, A. L. Alexander, and M. J. Sellars, Phys. Rev. B 74, 195101 (2006).

${ }^{22}$ R. Ahlefeldt, W. Hutchison, and M. Sellars, J. Lumin. 130, 1594 (2010).

${ }^{23}$ M. B. Schulz and C. D. Jeffries, Phys. Rev. 159, 277 (1967).

${ }^{24}$ J. Mlynek, N. C. Wong, R. G. DeVoe, E. S. Kintzer, and R. G. Brewer, Phys. Rev. Lett. 50, 993 (1983).

${ }^{25}$ A. Habenschuss and F. H. Spedding, Cryst. Struct. Commun. 9, 213 (1980).

${ }^{26}$ A. Habenschuss and F. H. Spedding, Cryst. Struct. Commun. 9, 157 (1980).

${ }^{27}$ A. Habenschuss and F. H. Spedding, Cryst. Struct. Commun. 9, 71 (1980).

${ }^{28}$ R. J. Birgeneau, Appl. Phys. Lett. 13, 193 (1968).

${ }^{29}$ W. Fricke, Z. Phys. B 33, 255 (1979). 